Horizons philosophiques

Horizons

\title{
Spiritualité, authenticité et l'expérience ordinaire : la figure d'Emerson dans Les Sources du Moi de Taylor
}

\section{Stéphane Bastien}

Volume 13, numéro 1, automne 2002

Religion et pluralisme

URI : https://id.erudit.org/iderudit/801220ar

DOI : https://doi.org/10.7202/801220ar

Aller au sommaire du numéro

Éditeur(s)

Collège Édouard-Montpetit

ISSN

1181-9227 (imprimé)

1920-2954 (numérique)

Découvrir la revue

Citer cet article

Bastien, S. (2002). Spiritualité, authenticité et l'expérience ordinaire : la figure d'Emerson dans Les Sources du Moi de Taylor. Horizons philosophiques, 13(1), 13-25. https://doi.org/10.7202/801220ar d'utilisation que vous pouvez consulter en ligne. 


\title{
SPIRITUALITÉ, AUTHENTICITÉ ET L'EXPÉRIENCE ORDINAIRE : LA FIGURE D'EMERSON DANS LES SOURCES DU MOI DE TAYLOR
}

\author{
Emerson possède cette bonne et spirituelle sérénité \\ qui décourage tout sérieux; il ne sait absolument pas \\ combien il est déjà vieux et combien il sera encore \\ jeune... Son esprit trouve toujours des raisons d'être \\ heureux et même reconnaissant... Friedrich \\ Nietzsche ${ }^{1}$ \\ It is said that Emerson is not a philosopher. I find this \\ denegation false or true as it is said in blame or praise \\ (...). When the critic writes of a lack of method, of \\ absence of continuity, of a coherent logic... the critic, \\ to my mind, but writes down his own incapacity to \\ follow a logic that is finely wrought. John Dewey²
}

Dans Les Sources du Moi ${ }^{3}$, Charles Taylor nous propose une esquisse philosophique de l'évolution de l'identité moderne. Une telle esquisse est certes complexe et fait appel à une sélection minutieuse des thèmes traités et des philosophes étudiés. II y a pourtant certaines omissions dans le tableau de Taylor qui nous laissent perplexes. Entre autres, comme le fait remarquer Richard Rorty 4 , Taylor n'intègre pas l'apport important de John Dewey qui, de par sa reconstruction originale de la notion d'expérience, n'est assimilable ni au courant des Lumières, ni non plus, au scientisme naïf du dixneuvième siècle ou encore, selon nous, à cette hégémonie de la

1. Friedrich Nietzsche, Le crépuscule des idoles, Paris, GF-Flammarion, 1985.

2. John Dewey, "Emerson, The Philosopher of Democracy", Collected Works : Middle Works, vol. 3, Carbondale, Southern Illinois University Press, 1976.

3. Charles Taylor, Les Sources du Moi. La formation de l'identité moderne, Québec, Boréal, 1998.

4. Richard Rorty, "Taylor on Self-Celebration and Gratitude", in Philosophy and Phenomenological Research, Buffalo, University of Buffalo for the International Phenomenological Society, 54 (1), 1994, p. 199. Dans le même numéro, Taylor rétorque en affirmant : "l'm not yet satisfied with the Deweyan constitutives goods", p. 213. Pour une analyse approfondie de ce débat, voir : Hans Joas, The Genesis of Value, Chicago, University of Chicago Press, 2000. 
rationalité instrumentale si critiquée par les penseurs heideggeriens. En revanche, il y a des inclusions qui peuvent surprendre, notamment celle de Ralph Waldo Emerson, dont la pensée est à la fois fortement spirituelle et pragmatique. En fait, le «transcendantalisme» d'Emerson se rapproche de façon convaincante de ce que Taylor nomme le "courant expressiviste". C'est pourquoi nous voulons montrer en quoi Emerson est une figure importante pour comprendre l'identité moderne ainsi que les sources morales et spirituelles qui animent la modernité. En fait, et c'est la thèse sous-jacente de cette recherche, Emerson représente un point focal où l'on retrouve, à des degrés divers, la majorité des grands idéaux modernes, ou idées-forces de la modernité, tels que Taylor les a développés dans Les Sources $d u$ Moi. Parmi ces idées-forces, mentionnons entre autres : l'affirmation de la vie ordinaire, la puissance créatrice (expressive) de l'être humain, une certaine conception du Transcendant ou de Dieu, une vision riche et complexe de l'individualité et, bien sûr, la quête de l'authenticité comme point culminant de l'expérience humaine. Ici, chacun de ces idéaux sera reconnu en tant que bien moderne (quoique pas nécessairement développé de manière égale tout au long du texte). Plus précisément, nous allons expliciter le rapport entre le courant expressiviste et l'idéal de l'authenticité, ainsi que la place qu'occupe Emerson dans le déploiement de ces idées-forces. II sera question, dans un premier temps, de démontrer que le tableau que brosse Taylor de la culture expressiviste peut nous mener à Emerson. Ensuite, nous tracerons brièvement la figure d'Emerson telle qu'elle apparaît dans les Sources du Moi. II sera enfin question de dégager ce que Taylor aurait pu ajouter concernant la place d'Emerson dans son tableau. Nous allons voir que les conceptions émersoniennes de la Confiance en soi (Self-reliance) et de L'Homme pensant (Man Thinking), débouchent sur une vision de l'authenticité et sur ce que Stanley Cavell appelle le perfectionnisme moral.

\section{I- De l'affirmation de soi à l'expression de soi :}

Au sujet d'Emerson, Cavell remarque : «Je ne connais personne dans l'histoire de la philosophie occidentale à qui l'on dénie de manière aussi obsessionnelle le titre de philosophe ${ }^{5}$ ». La figure d'Emerson est certes étrange en philosophie. Comme Montaigne et Nietzsche, il se veut non-systématique, faisant appel à l'essai comme forme d'expression, visant plutôt à réaliser un idéal de vie qu'un

5. Stanley Cavell, Statuts d'Emerson : Constitution, philosophie, politique; en appendice : Ralph Waldo Emerson, «Destin» et «Expérience», Paris, L'éclat, coll. «Tiré à part", 1992, p. 7. 
exposé d'idées claires et distinctes. Mais c'est bien parce qu'il est si difficile de le situer qu'il représente une occasion privilégiée pour méditer sur la modernité et peut-être apprendre encore quelque chose sur "la philosophie, sur ce qui la rend douloureuse 6 ». Toutefois, il convient d'abord de retracer le fil conducteur à l'intérieur même des Sources du Moi qui mène à Emerson et à l'idéal moderne de l'authenticité.

Pour Taylor, la modernité constitue, en elle-même, une culture presque à part entière, puisqu'elle présuppose un ensemble de biens ou une constellation de valeurs qui lui est propre. L'essor du commerce, la privatisation de la famille et du mariage, le statut de l'enfant et le sentiment de la nature, sont autant d'avenues qui nous permettent de saisir des aspects de l'identité moderne que le sont, par exemple, le développement de la rationalité scientifique instrumentale ou l'avènement de l'ère technologique. Plus encore, l'esprit moderne représente un passage (entamé par le christianisme) d'une certaine forme d'extériorité (logos ontique) à une plus grande intériorité. Cette nouvelle conception de l'intériorité est à la racine de l'affirmation de la vie ordinaire, la valorisation du sentiment et de l'expression de soi, ainsi que d'une nouvelle conception du Transcendant.

L'élan individualiste si caractéristique de la modernité s'est manifesté graduellement à travers toutes les structures sociales, quitte à les faire éclater entièrement à l'occasion. À cet égard, nos conceptions de l'enfance et de la famille sont des références constitutives pour comprendre la valorisation de la vie ordinaire et l'importance que l'on accorde à l'expression personnelle. En effet, avec la modernité, le couple sera fondé davantage sur l'idéal de l'amour. L'ancien contrôle socioculturel de la famille a cédé, au sein même de la modernité, à une conscience de l'intimité où le mariage est libre et alimenté par l'affection, ainsi que par les affinités naturelles entre les individus. Mais du même coup, remarque Taylor, «elle élimina le monde ouvert, semblable à un aquarium, de la société traditionnelle7", c'est-à-dire que le monde "public» fut remplacé par le monde "privé" de l'individu, reconnaissance à laquelle même l'enfant a droit. Depuis, l'enfance "revêt une identité en tant que phase distincte du cycle de la vie, dotée de sentiments particuliers et de besoins propres ${ }^{8}$ ». Cette perspective est radicalement différente de la conception antique aristotélicienne qui comprenait l'enfance comme
6. Ibid.
7. C. Taylor, op.cit., p.373.
8. Ibid. 
une simple transition vers l'âge adulte, d'un état inférieur et incomplet à un état de complétude. Mais plus encore, c'est dans la relation entre l'individu et le sentiment de la nature que Taylor identifie l'une des expériences saillantes de la modernité.

Du point de vue d'une conception élargie de l'esprit moderne, la nature n'est plus cet ordre extrinsèque dans lequel nous devons trouver notre place comme le pion dans un vaste jeu d'échec. Dorénavant, le monde est le flux hétérogène de l'expérience immédiate où «nous éprouvons la nature de façon paradigmatique et centrale, non pas dans une vision de l'ordre, mais en éprouvant l'impulsion intérieure juste ${ }^{\text {". }}$. Or, chez les romantiques cette impulsion prend la forme d'une "voix" qui nous guide et nous renseigne sur nous-mêmes. Toutefois, cette voix intérieure, qui est le mode d'accès privilégié pour saisir la nature dans son essence, peut être aliénée, étouffée : "et ce qui peut l'étouffer, c'est précisément l'attitude désengagée de la raison calculatrice, qui perçoit la nature de l'extérieur comme un ordre simplement observé10". En d'autres mots, la nature sollicite, de notre part, une forme d'action, notamment la capacité d'entrer en rapport avec elle et avec sa voix en nous. Ce rapport doit s'établir, non par la pure rationalité désengagée, mais par un acte intérieur : «Être en accord avec la nature, c'est faire l'expérience de la richesse, de la plénitude et de la signification de ces désirs - répondre au courant de vie dans la nature 11 ». De par sa dimension "active», plutôt que " contemplative", cette pulsion intérieure peut également être comprise "comme un enchaînement de choses exigeant des actions", perspective que nous pouvons retracer, poursuit Taylor, "au transcendantalisme mais aussi au pragmatisme ${ }^{12}$ ". Chacun ici est appelé à participer dans le déploiement de la nature par l'entremise de son individualité profonde et non en suivant un mode préétabli de comportement qui serait extérieur à notre nature : «Nous ne devons pas espérer trouver nos modèles au dehors ${ }^{13}$ ". Ou dans les mots du sage barde, Walt Whitman : "Votre route, ce n'est pas à moi, mais à vous, à personne d'autre que vous de la parcourir...14». Et que ce soit Descartes qui nous invite à trouver une méthode ou Rousseau qui médite en promeneur solitaire, classique et romantique se rencontrent dans cette affirmation de la personne.

9. Ibid., p. 364.

10. Ibid.

11. Ibid., p. 466-467.

12. Ibid., p. 300.

13. Ibid., p. 471.

14. Walt Whitman, "C'est moi que je célèbre", in Feuilles d'herbes, Paris, Bernard Grasset, coll. "Cahier rouge", 1989, sec. 46 (p.95). 
La valorisation de l'individualité atteint son paroxysme avec la découverte que le plus intime en nous débouche, tôt ou tard, sur ce qu'il y a de plus universel. Toutefois, chacun doit trouver sa manière propre de réaliser l'universel car «tout comme les manifestations du grand courant de la vie dans le reste de la nature ne peuvent être les mêmes que sa réalisation dans la vie humaine, sa réalisation en vous peut différer de sa réalisation en moi ${ }^{15}$ ». L'individualité, en tant que valeur moderne, a donc évolué de l'affirmation de soi et de la vie ordinaire à l'expression de soi. En ce sens, l'art expressiviste n'est que cette propension vers l'actualisation des potentialités humaines, une incitation à devenir "l'artiste de sa vie" ou pour employer un autre vocabulaire, être notre propre projet.

Cette visée individualisante, quoiqu'elle occasionne une certaine rupture d'avec l'ordre ancien du logos ontique d'une part, et d'autre part se montre souvent réfractaire devant la tendance matérialisante de la science moderne, poursuit tout de même une tentative de réconciliation avec ces dimensions de l'expérience, cherchant une synthèse entre la volonté d'exploration de soi et la maîtrise de la nature : «'expressivisme met en rapport deux territoires autrement que le faisait l'humanisme des Lumières, en cela que l'exploration du moi et celle de la nature peuvent être menées concurremment ${ }^{16}$ ». Ce qui donne raison à Emerson pour qui chez l'Homme pensant (Man Thinking), «le précepte ancien, connais-toi toi-même, et le précepte moderne étudie la nature, se rejoignent enfin ${ }^{17}$ ». Si Emerson penche plutôt du côté d'une pensée poétique, ce n'est pas parce qu'il est contre la science mais bien, comme nous allons le voir, par souci d'une philosophie qui interpelle l'être entier.

Ainsi, nous allons voir que les deux territoires d'exploration morale qui caractérisent la modernité selon Taylor, c'est-à-dire la raison instrumentale et la nature comme source, occupent une place prépondérante dans la pensée d'Emerson. Mais loin de constituer une simple figure romantique, nous serons amenés à percevoir chez Emerson une certaine tension caractéristique de la modernité, tension entre l'expression libre et les contraintes du réel. En ce sens, Emerson annonce aussi bien Nietzsche et le postromantisme que le pragmatisme.

15. Taylor, op.cit., p. 471.

16. Ibid., p. 489.

17. Ralph Waldo Emerson, L'intellectuel américain, Québec, Loup de Gouttière, coll. "Le lieu du loup", 1992, p. 76. 


\section{II- La figure d'Emerson :}

II importe d'abord de noter que la place allouée à Emerson dans Les Sources du Moi est relativement restreinte si on compare celle-ci à l'exposé élaboré consacré à Montaigne ou encore à la discussion soutenue sur les idées de Nietzsche. Pourtant, Emerson, accompagné de Whitman, est mentionné à plus de cinq reprises, ce qui est déjà plus significatif que l'omission complète de John Dewey ou William James ${ }^{18}$. Mais mon but n'est ni de défendre la philosophie américaine, ni même le pragmatisme. Cependant, la question reste à savoir pourquoi Emerson mérite d'être mentionné aux yeux de Taylor? Une partie de la réponse nous a été fournie par notre discussion sur le tournant expressiviste et l'individualisme moderne.

En effet, Emerson est bel et bien le penseur de l'individualisme dans le sens où l'entend Taylor, c'est-à-dire d'une individualité qui, comme chez Kant mais aussi comme chez les romantiques, est porteuse d'universalité. À cet égard, les propos d'Emerson dans son essai célèbre Self-Reliance (La Confiance en soi) me semblent particulièrement justes :

Croire en votre propre pensée, croire que ce qui est vrai pour vous au plus secret de votre cœur est vrai pour tous les hommes - c'est là le génie. Exprimez votre conviction profonde, et son sens en deviendra universel; car le moment venu, ce qui est le plus secret devient le plus répandu et notre pensée première nous est renvoyée par les trompettes du Jugement dernier ${ }^{19}$.

C'est là un passage puissant pour certains, et peut-être trop simpliste pour d'autres. Mais il reflète parfaitement cet effort typiquement "expressiviste" pour surmonter ce que Taylor nomme les horizons fragmentés de la vision moderne, en comprenant l'individualité comme rencontre entre le particulier et l'universel. Ici, l'expression la plus intime de la personne est une manifestation de l'universel. Pour Emerson, l'Étre (si l'on peut donner provisoirement ce nom à l'universel ou le courant de vie qui nous traverse) travaille à travers chacun de nous. C'est en ce sens que Taylor peut dire d'Emerson qu'il «erra aux frontières où se touchaient le théisme, le panthéisme et

18. II convient de mentionner que le dernier livre de Taylor porte justement sur la question de la religion chez James. Cf. Charles Taylor, Varieties of Religion Today: William James Revisited, Cambridge, Harvard University Press, 2002.

19. Ralph Waldo Emerson, La Confiance en soi et autres essais, Paris, Rivages poche, coll. «Petite bibliothèque», 2000, p. 85-86. 
le non-théisme 20 ». Emerson retient une conception forte du Divin mais cherche toujours à dégager la pureté de cette expérience de ses oripeaux dogmatiques, préférant les paysages naturels et les événements de la vie ordinaire aux grandes révélations d'Église : "J'embrasse le commun; j'explore le familier, le simple; je m'assois à leurs pieds. Ouvrez-moi des perspectives sur aujourd'hui et je vous laisse les mondes antiques et futurs ${ }^{21}$ ». Le religieux, pour Emerson, se retrouve tout autant dans la vie quotidienne que dans les grands événements de l'histoire. Tout ce qu'il réclame d'une âme profonde, c'est qu'elle lui montre que "la plus grande cause spirituelle hante même, de sa sublime présence, ces faubourgs et ces lisières de la nature22». Ainsi, pour Emerson, tout comme pour Henry David Thoreau, la transcendance passe par la «transdescendance». Le plus élevé s'incarne dans le commun, l'ordinaire; les profondeurs de l'âme humaine correspondent à la nature divine en nous.

Taylor observe, à juste titre selon nous, que les objectifs du transcendantalisme vont de pair avec ceux du tournant expressiviste : "Les objectifs sont l'expression de soi, la réalisation de soi, l'accomplissement de soi, la découverte de l'authenticité23". Et si le mouvement du "potentiel humain", qui s'inspire entre autres du transcendantalisme, fait parfois fausse route en proposant des psychologies postfreudiennes qui "escamotent fréquemment le sentiment tragique du conflit qui était essentiel à la pensée de Freud ${ }^{24}$ », nous allons voir qu'il en va tout autrement chez Emerson qui porte en lui les idéaux de la modernité, ainsi que les tensions naturelles qui les accompagnent. Ceux-ci incluent du même coup ce moment de rupture où l'individu devient conscient de ses limites et d'un écart entre la pensée et le monde, moment que l'on peut qualifier de "postmoderne", mais qui n'en demeure pas moins intégrable à la perspective moderne et à la vision expressiviste.

II faut encore noter qu'Emerson se considère lui-même, à l'instar de Montaigne, comme un expérimentateur («l am only an experimenter25»); thème exploité avec subtilité par Nietzsche, lecteur assidu de l'un comme de l'autre, lorsqu'il annonce qu'une «nouvelle race de philosophes monte à l'horizon... ces philosophes de l'avenir

20. Taylor, op.cit.,p. 512.

21. Emerson, L'intellectuel américain, p. 94.

22. Ibid., p. 95.

23. Taylor, op.cit., p. 632.

24. Ibid., p. 620.

25. Ralph Waldo Emerson, "Circles", in Selected Writings, New York, Penguin, coll. "Signet Classic", 1983, p. 288. 
voudraient avoir le droit, peut-être même le tort, d'être appelés des tentateurs ${ }^{26}$ ". Toutefois, cette nouvelle race de penseurs n'est pas celle de l'avenir, mais bien une race de philosophes typiquement modernes, peut-être «trop modernes". En tant qu'héritier de cette vision expressiviste, Emerson incarne donc la quête de l'authenticité comme la plus centrale des préoccupations philosophiques. Mais celle-ci va de pair avec une certaine tension tragique entre le penseur et la société dans laquelle il vit. Cependant, pour Emerson, comme pour Taylor, ce conflit peut également être la pulsion nécessaire vers une authenticité plus globale, intégrant dans un même élan l'expression de soi, la reconnaissance de l'autre et la connaissance de la Nature.

\section{III- Authenticité et perfectionnisme :}

Parmi les valeurs de la modernité, il faut compter l'idéal d'authenticité qui englobe chez Emerson, tout comme pour Taylor, la connaissance de soi, l'expression de soi et la réalisation de soi. Toutefois, Emerson parle plutôt de Self-Reliance, que l'on traduit habituellement par confiance en soi, mais qui se rapproche davantage de l'idée d'autonomie. Cependant, les deux termes me semblent compatibles et se rapportent à notre capacité d'être vrai envers soi-même. "Votre action authentique se justifiera d'elle-même et justifiera vos autres actions authentiques 27 ", déclare le sage de Concord. Et Taylor remarque : "Si être authentique, c'est être sincère avec soi-même, recouvrer son propre sentiment d'existence, nous ne pourrons alors y parvenir pleinement qu'en reconnaissant que ce sentiment nous relie à un tout plus vaste ${ }^{28}$ ".

Or la confiance en soi chez Emerson ainsi que chez les romantiques, se rattache à notre faculté intérieure de prendre contact avec le courant de vie dans la nature : "Nous nous trouvons dans les bras d'une immense intelligence qui fait de nous les récepteurs de sa vérité et les organes de son activité 29 ", écrit Emerson. La confiance en

26. Friedrich Nietzsche, Par-delà le bien et le mal, Paris, Gallimard, coll. "Folio Essais", 1998, aphorisme 42. Sur la relation entre Nietzsche et Emerson, voir l'étude de George J. Stack (Nietzsche and Emerson : An Elective Affinity, Athens, Ohio University Press, 1992), ainsi que l'essai de Régis Michaud («Emerson et Nietzsche", in Autour d'Emerson, Paris, Bossard, 1924).

27. Emerson, La Confiance en soi et autres essais, p. 98.

28. Charles Taylor, Grandeur et misère de la modernité, Québec, Bellarmin, coll. "L'essentiel", 1992, p. 115.

29. Emerson, La Confiance en soi et autres essais, p. 104. II convient de mentionner que c'est à ce sentiment d'être englobé par un utout plus vaste» que Umberto Eco, suivant Dewey, reconnaît l'une des caractéristiques de l'expérience esthétique : 
soi est la racine véritable de l'autonomie qui, dans son sens profond, suggère un passage de l'état de minorité à l'état de majorité, pour reprendre la formule kantienne, mais sans la perte de ce qui nous rend unique. C'est pourquoi Emerson comprend l'autonomie comme la volonté d'être soi au sein même de la foule : "ll est facile, étant dans le monde, de vivre selon l'opinion du monde; il est facile, dans la solitude, de vivre selon la nôtre, mais il a de la grandeur, celui qui au milieu de la foule garde avec une suavité parfaite l'indépendance de la solitude ${ }^{30}$ ». L'Homme pensant démontre de l'indépendance dans ses jugements; il est celui ou celle qui sait distinguer ce qui vaut. D'où la nécessité de la méditation philosophique, cet art qui, avant même l'expression de soi, "consiste à choisir les objets qui ont du relief 31 ». Thème essentiel, selon Cavell, reliant les vertus de l'Homme pensant d'Emerson à la notion de l'Être pensant chez Heidegger, par l'entremise de Nietzsche ${ }^{32}$. En effet, comme Heidegger plus tard, Emerson nous invite à une pensée méditante qu'il oppose farouchement, non pas à la dimension pragmatique de notre existence, mais à l'entendement désengagé qui suppute au détriment de la qualité du moment.

C'est en ce sens que le désir d'authenticité peut entrer en conflit avec la difficulté de comprendre le monde qui nous entoure et de vivre avec l'autre. Nous ne vivons pas dans un vacuum, et l'actualisation de soi n'a de sens que dans et avec le monde qui nous entoure. En fait, remarque Taylor, «aucun accomplissement n'aurait de valeur dans un monde où littéralement rien n'aurait d'importance que l'accomplissement personnel33". C'est parce que nous participons à un monde, que nous sommes des êtres dans le monde, que la réalisation personnelle peut avoir un sens et une valeur, pour soi-même et pour autrui.

Mais l'altérité implicite qui subsiste entre le désir d'être soi et la société rend l'actualisation personnelle parfois pénible et complexe. II

“Or, pour Dewey, le propre de l'art serait précisément d'évoquer et d'accentuer "cette faculté d'être un tout, d'appartenir à un tout plus grand qui inclut toute chose et qui n'est autre que l'univers dans lequel nous vivons"; de là l'émotion religieuse que provoque en nous la contemplation esthétique", L'œuvre ouverte, Paris, Seuil, coll. "Points", 1965, p.45. Nous abordons plus loin la question de l'esthétique pragmatique chez Emerson.

30. Ibid., p. 93.

31. Ralph Waldo Emerson, "La conduite de la vie», in Pages choisies, Paris, Astra, 1976, p. 256.

32. Sur la relation entre Heidegger et Emerson, voir Stanley Cavell, "La pensée de l'aversion : Représentations émersoniennes chez Heidegger et Nietzsche", in Conditions nobles et ignobles : La constitution du perfectionnisme moral émersonien, Paris, L'éclat, coll. «Tiré à part», 1993. Et sur le thème de l'Être pensant, voir Martin Heidegger, "Sérénité», in Question III, Paris, Gallimard, 1961.

33. Taylor, les sources du moi, p. 633. 
y a toujours "le monde... cet autre moi»34, note Emerson, avec lequel je dois composer et dans lequel je pense. Et trop souvent, «je sais que le monde avec lequel je parle dans la ville et dans les fermes n'est pas le monde que je pense 35 ". II y a donc discordance tout autant que concordance entre le penseur et le monde, et quiconque recherche l'authenticité se voit confronté à ce qu'Emerson nomme les seigneurs de la vie : "'llllusion, le Tempérament, la Succession, la Surface, la Surprise, la Réalité, la Subjectivité36». Ce sont là les obstacles qui nous empêchent de nous voir tels que nous sommes et d'accéder réellement à qui nous sommes. Nous pouvons être dupes de nous-mêmes, ignorer les motivations réelles qui nous guident et ainsi saboter notre processus d'individuation, tout en croyant atteindre le summum de l'accomplissement personnel et social.

Emerson est donc loin d'un idéalisme naîf qui escamote la dimension tragique de la vie. Au plus, il est «mélioriste», pour reprendre l'expression de William James. En d'autres mots, pour Emerson, le monde est une toile de circonstances ou de contextes, qui laisse place à l'amélioration et au perfectionnement. L'authenticité réside dans ma capacité à transformer les matériaux de l'expérience en une $œ u v r e$ d'art, l'œuvre que peut devenir ma vie. Mais cette œuvre doit intégrer la souffrance et le conflit. Le destin n'est qu'un mot que nous attribuons à toutes les circonstances qui s'opposent à notre volonté immédiate : «Tout ce qui nous limite, nous l'appelons Destin 37». Cette dissonance entre l'idéal et le réel est la véritable source du désenchantement de la vie que l'on ressent dans la démarche "postromantique" à la Schopenhauer dont la vision, nous dit Taylor, "peut nous soulager du poids excessif de l'optimisme schillérien, qui nous écrase sous le sentiment de notre propre insuffisance ${ }^{38}$ ". L'exigence de l'authenticité, comme l'exigence de la confiance en soi, va de concert avec la reconnaissance du poids existentiel de nos limites et de nos faiblesses.

Pourtant, cela ne signifie nullement que l'idéal de l'authenticité soit faux ou même contradictoire. II implique certes un paradoxe, mais celui-ci doit être intégré dans une quête globale capable d'exprimer toutes les facettes de la nature humaine. À cet égard, la pensée d'Emerson expose la complexité de la réalité humaine, mais elle fait aussi preuve d'une confiance indéniable envers les capacités

34. Emerson, L'intellectuel américain, p. 82.

35. Emerson, «Expérience», p. 94.

36. Ibid., p. 93.

37. Emerson, «Destin», p. 52. 
spirituelles de l'homme : "Un homme doit chevaucher alternativement sa nature privée et sa nature publique, comme au cirque les acrobates se jettent souplement de cheval en cheval, ou bien plantent un pied sur le dos d'un cheval et l'autre pied sur un autre ${ }^{39}$ ”. C'est donc reconnaître que l'expression et la réalisation de soi doivent relier tout à la fois ma nature privée et ma nature publique. Et tel que le note Taylor : "L'idéal de l'authenticité inclut certains concepts de société, une idée, en tout cas, de la façon dont les gens doivent vivre ensemble 40 ". Ce "vivre ensemble» n'est rien de moins que la tâche de l'éthique qui est, selon la définition qu'en donne Paul Ricoeur, «la visée de la vie bonne avec et pour autrui...41». Cette visée éthique doit inclure un certain idéal de vie authentique, sinon tout effort d'une société juste risque de s'effondrer ou de dégénérer dans de simples rapports économiques et politiques, négligeant par là même la source morale de la justice.

Ainsi, la sphère publique et la sphère privée convergent dans l'idéal d'authenticité et présupposent pour Emerson, la perfectibilité de la nature humaine : "an Emersonian perfectionism that insists on continuous self-improvement", souligne Richard Shusterman 42. Toutefois, comme le rappelle Cavell, il faut se prémunir contre des interprétations faciles du perfectionnisme émersonnien : «Les versions fausses ou dégradées du perfectionnisme semblent être partout de nos jours, depuis les livres à gros tirage portant des titres du style Pour s'aimer soi-même, jusqu'à la campagne de publicité pour l'Armée de terre à la télévision encourageant à s'engager avec le slogan : "Réalisez-vous complètement"43".

Qu'est-ce donc qui différencie Emerson de ses pasticheurs superficiels? D'abord, de toute évidence, le recrutement pour l'Armée américaine n'a rien de ce que le sage de Concord associerait à l'autonomie, puisque dans ce cas-ci il est moins question de devenir soi-même que d'acquérir une identité faite sur mesure, celle entre autres qui fera de nous des «mercenaires»44. Ensuite, les livres de

38. Taylor, les sources du moi, p.553.

39. Ibid., p. 67.

40. Taylor, Grandeur et misère de la modemité, p. 61.

41. Paul Ricœur, Soi-même comme un autre, Paris, Seuil, coll. «Points Essais», 1996, 7 ième étude, p. 202.

42. Richard Shusterman, "Emerson's Pragmatist Aesthetics", in Revue internationale de philosophie, Bruxelles, 53 (1), 1999, p. 94.

43. Cavell, Conditions nobles et ignobles: la constitution du perfectionnisme moral émersonien, p. 66.

44. Ibid. 
psychologie et de spiritualité populaires ne font pas toujours preuve du profond questionnement existentiel qui occupe les écrits d'Emerson, tel que nous l'avons démontré jusqu'ici. Pour Cavell, le perfectionnisme consiste moins «à réfréner le mal qu'à libérer le bien, à le libérer d'un sentiment de désespoir du bien (du bien et du mal en chacun de nous) ${ }^{45}$ ». Ou, pour dire la même chose autrement, il nous invite à désespérer du désespoir qui nous empêche d'accéder à notre génie, pour reprendre Emerson, de renouer avec le bien en nous.

Ce serait donc une grave erreur de croire qu'il faille se laisser enliser soit dans un optimisme béat, soit dans un pessimisme de l'absurde. Chez Emerson la réflexion philosophique n'est toujours qu'une préparation à la vie bonne. "Je ne sais rien de plus encourageant (note Thoreau) que l'aptitude incontestable de l'homme à élever sa vie grâce à un effort conscient 46 ». Cependant, cette aptitude doit être cultivée. Et comme l'explique Shusterman, chez Emerson, comme chez Dewey d'ailleurs, l'art possède une visée éthique : "Art is not for its own sake but for the sake of better living

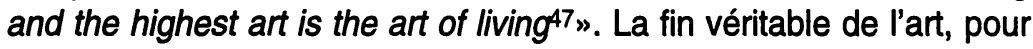
Emerson, c'est l'art de vivre et cle roman vrai que le monde existe pour réaliser, sera la transformation du génie en pouvoir pratique ${ }^{48}$ ". Les idées et les idéaux émergent de l'expérience intime du poète et du philosophe, mais ils ont comme tâche de retourner dans l'expérience partagée, intersubjective, c'est-à-dire démocratique. Non pas pour être adoptés aveuglément comme un message venant d'un arrière monde, mais dans l'optique d'inspirer et d'orienter l'action, incluant les débats et les critiques qu'ils peuvent susciter dans les rapports humains. Et c'est enfin à l'intérieur du tissu des interactions humaines qu'un principe de vie doit acquérir ses lettres de noblesse.

Bref, la créativité humaine ne se déploie pas dans un vide mais au cœur de nos relations dans et avec le monde, et le véritable but de la réalisation et de la création de soi n'est autre que la transformation des conditions actuelles de l'existence en des perspectives nouvelles. L'expérience esthétique, pour Emerson, tout comme pour Dewey, a une portée vitale : «il est pour l'Art un travail plus grand que les arts...

45. Ibid., p. 68.

46. Henry David Thoreau, Walden ou la vie dans les bois, Paris, Gallimard, coll. "L'imaginaire", 1990, p. 89.

47. Shusterman, art.cit., p. 89. Cf. John Dewey, Art as Experience, in The Collected Works: Later Works, Carbondale, Southern Illinois University Press, vol. 10, 1987. C'est dans ce texte que Dewey expose clairement sa conception de l'expérience esthétique.

48. Emerson, «Expérience», p. 94. 
sa fin n'est rien de moins que la création de l'homme et de la nature 49 ». Le fait que nous soyons des êtres dans le monde ne constitue donc pas une limite à notre épanouissement mais une condition de possibilité de celui-ci, un horizon de sens existentiel et interpersonnel.

\section{Remarques finales :}

J'ai montré comment la figure d'Emerson dans Les Sources du Moi joue un rôle d'importance afin de mieux saisir l'identité moderne. Si Taylor ne consacre pas à Emerson une place prépondérante dans son exposé, il n'en demeure pas moins qu'Emerson incarne la majorité des grands thèmes de la formation de la modernité dans sa complexité. Celle-ci inclut le sentiment postromantique, voire postmoderne, du désenchantement du monde. Si nous prenons Taylor au sérieux lorsqu'il proclame la nécessité d'une réconciliation avec les origines de la modernité, alors je crois que les essais d'Emerson sont un échelon primordial dans notre ascension vers une autocompréhension de soi plus globale, ainsi qu'une manière de comprendre la situation de la philosophie aujourd'hui. De plus, notre interprétation d'Emerson, à cet égard, suggère en quoi le pragmatisme est porteur d'une forme d'épiphanie qui est en relation directe avec les deux territoires de la modernité. Enfin, retenons que la poussée première et l'aboutissement final de l'expérience esthétique et de l'expérience spirituelle chez Emerson, sont l'actualisation des potentialités humaines vers la croissance, la créativité et l'harmonie, avec et pour autrui, en vue d'une expérience plénière. Rien de moins, donc, que l'idéal de l'authenticité. 50

\section{Stéphane Bastien \\ Université d'Ottawa}

49. Emerson, "L'Art», in Pages choisies, p. 263.

50. Je désire remercier Daniel Tanguay pour ses commentaires lors de la première ébauche de ce texte. J'ai par la suite profité des conseils de Vance Mendenhall et des révisions de Geneviève Allard. 